\title{
Coking Prediction in Catalytic Glucose Conversion to Levulinic Acid Using Improved Lattice Boltzmann Model
}

Siwei Liu ${ }^{a, b, c, d}$, Xiangqian Wei, Weitao Sun ${ }^{e}$, Chenguang Wanga,b,c, Wenzhi Li ${ }^{e}$,

Longlong Ma ${ }^{a, b, c * *}$ Qiying Liu ${ }^{a, b, c, *}$

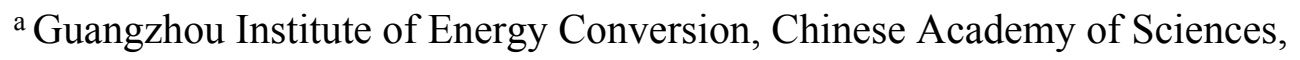
Guangzhou 510640, PR China

${ }^{\mathrm{b}}$ Key Laboratory of Renewable Energy, Chinese Academy of Sciences, Guangzhou 510640, PR China

${ }^{c}$ Guangdong Key Laboratory of New and Renewable Energy Research and Development, Guangzhou 510640, PR China

d University of Chinese Academy of Sciences, Beijing 100049, PR China

${ }^{\mathrm{e}}$ Laboratory of Basic Research in Biomass Conversion and Utilization, Department of Thermal Science and Energy Engineering, University of Science and Technology of China, Hefei 230026, PR China

*Corresponding author

E-mail address: liuqy@ms.giec.ac.cn (Qiying Liu)

**Co-Corresponding author

E-mail address: mall@ms.giec.ac.cn (Longlong Ma) 


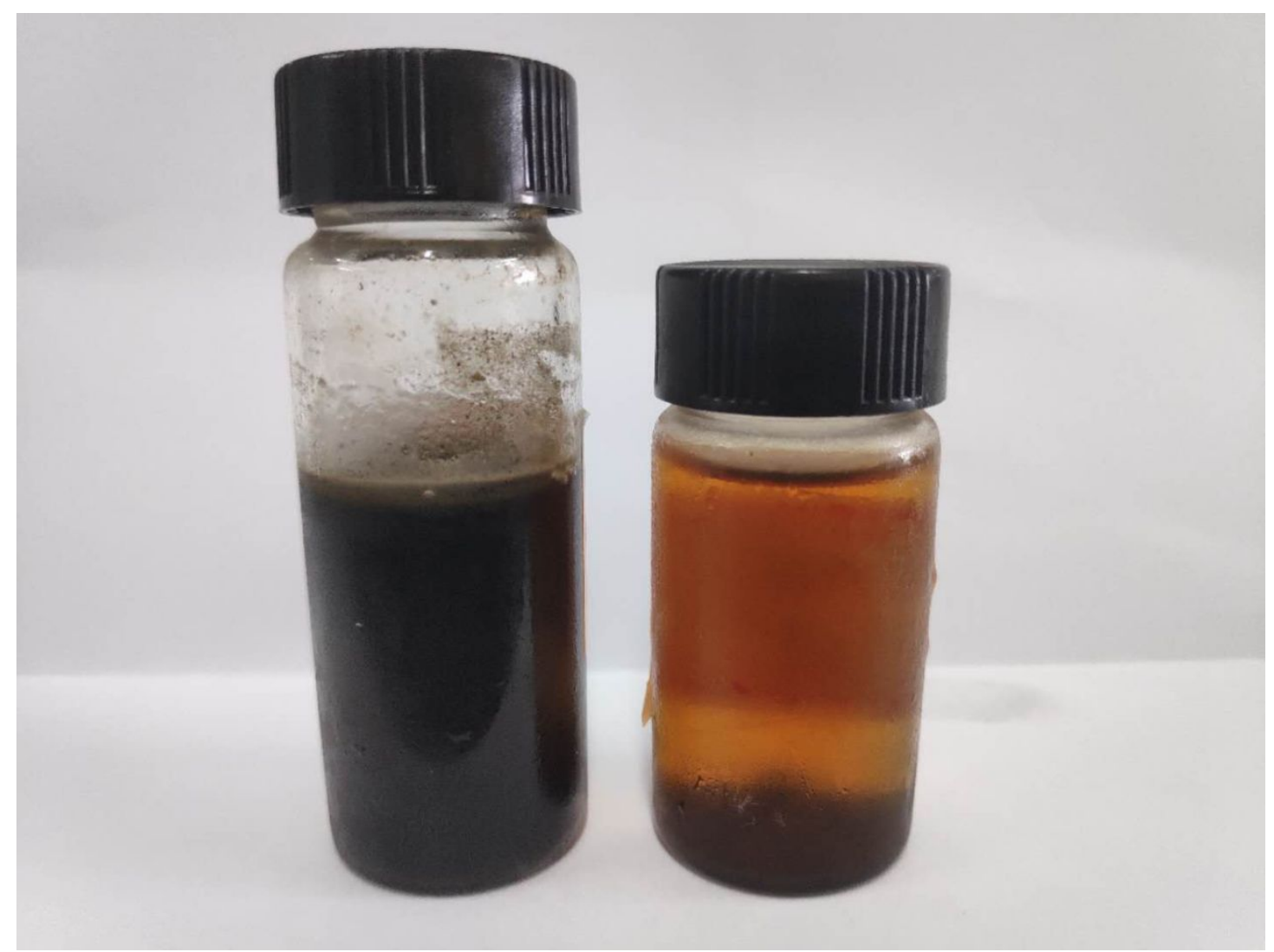

Figure S1. Comparison between the non-catalyzed coking and Fe/HY-catalyzed coking for glucose conversion at $180{ }^{\circ} \mathrm{C}$ for $3 \mathrm{~h}$. (Left: non-catalyzed coking; Right: Fe/HYcatalyzed coking) 


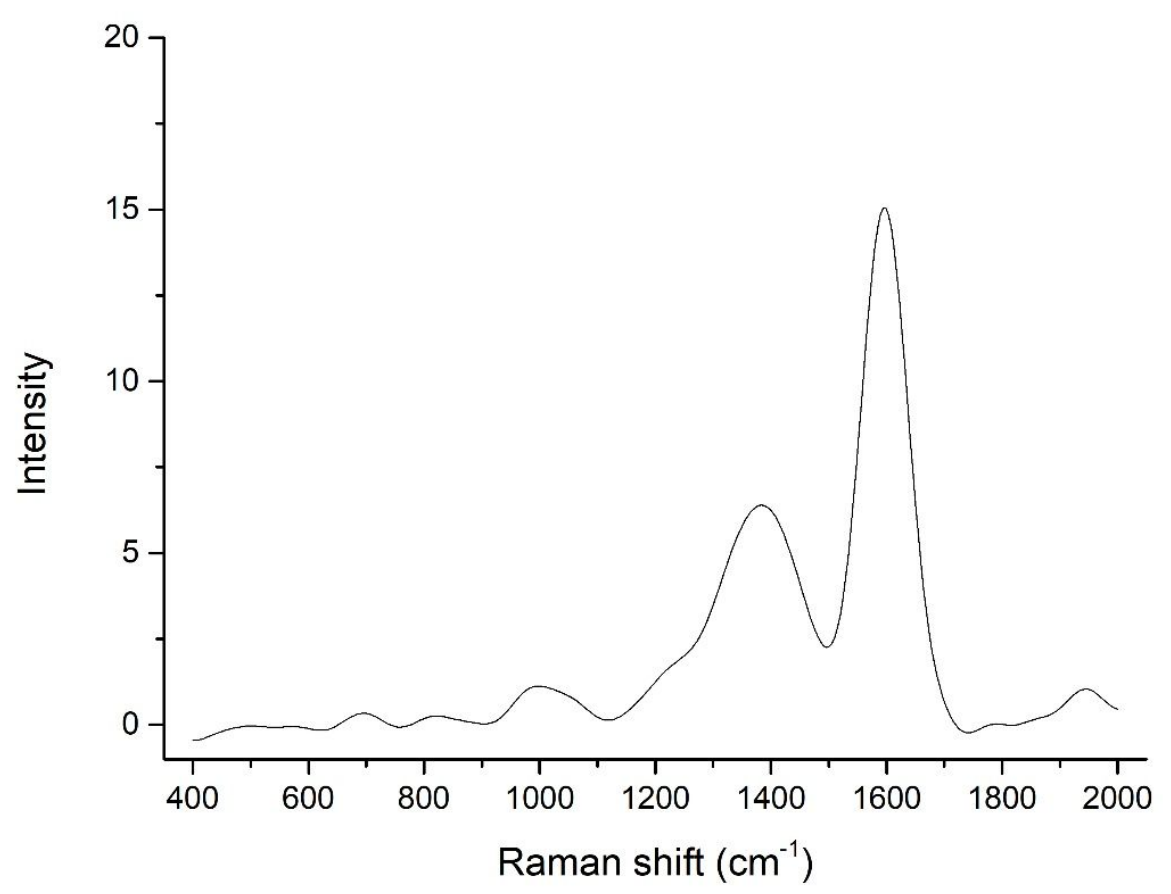

Figure S2. Raman spectra of coked catalyst after the reaction time of $3 \mathrm{~h}$ at $200{ }^{\circ} \mathrm{C}$. 


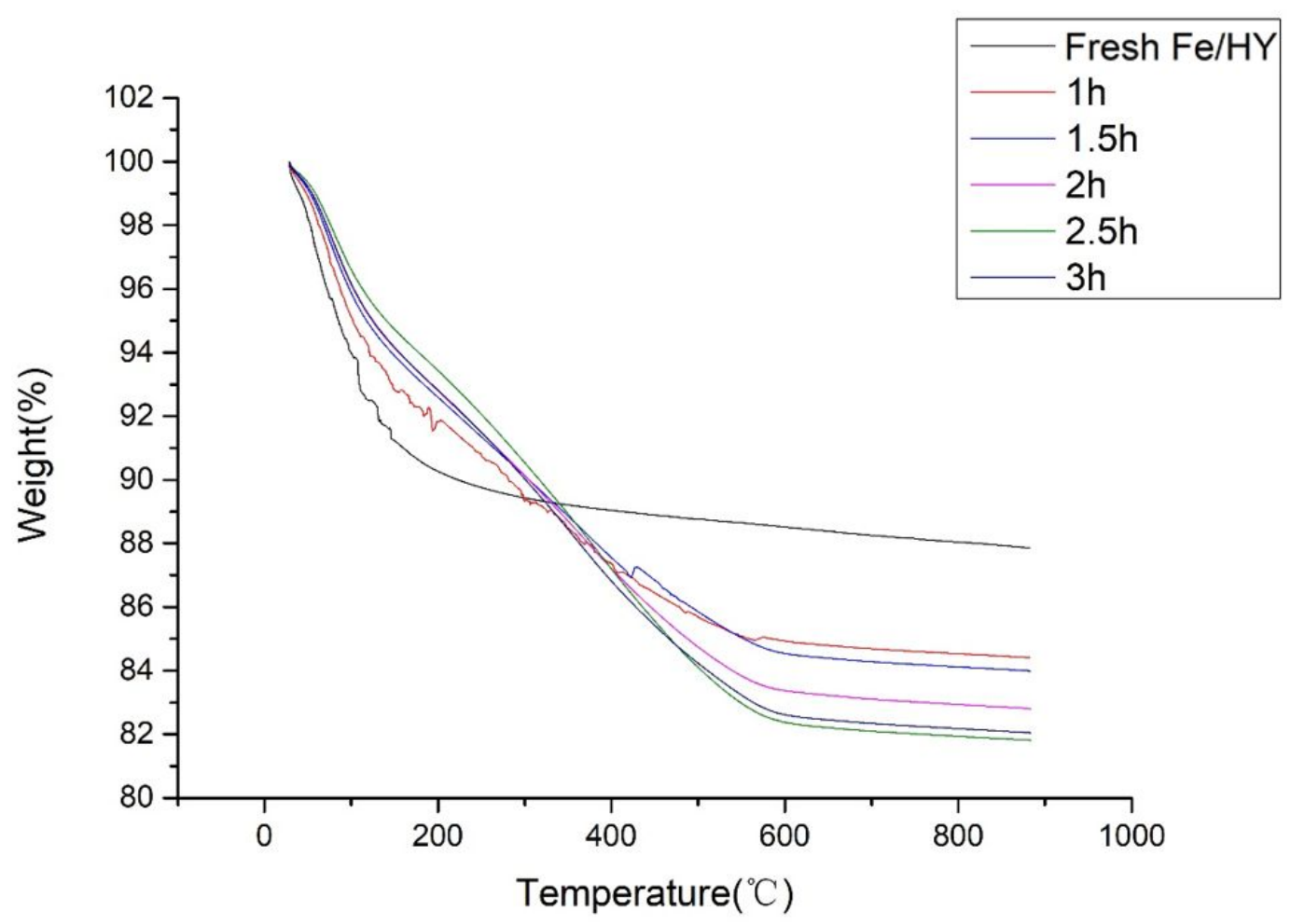

(a)

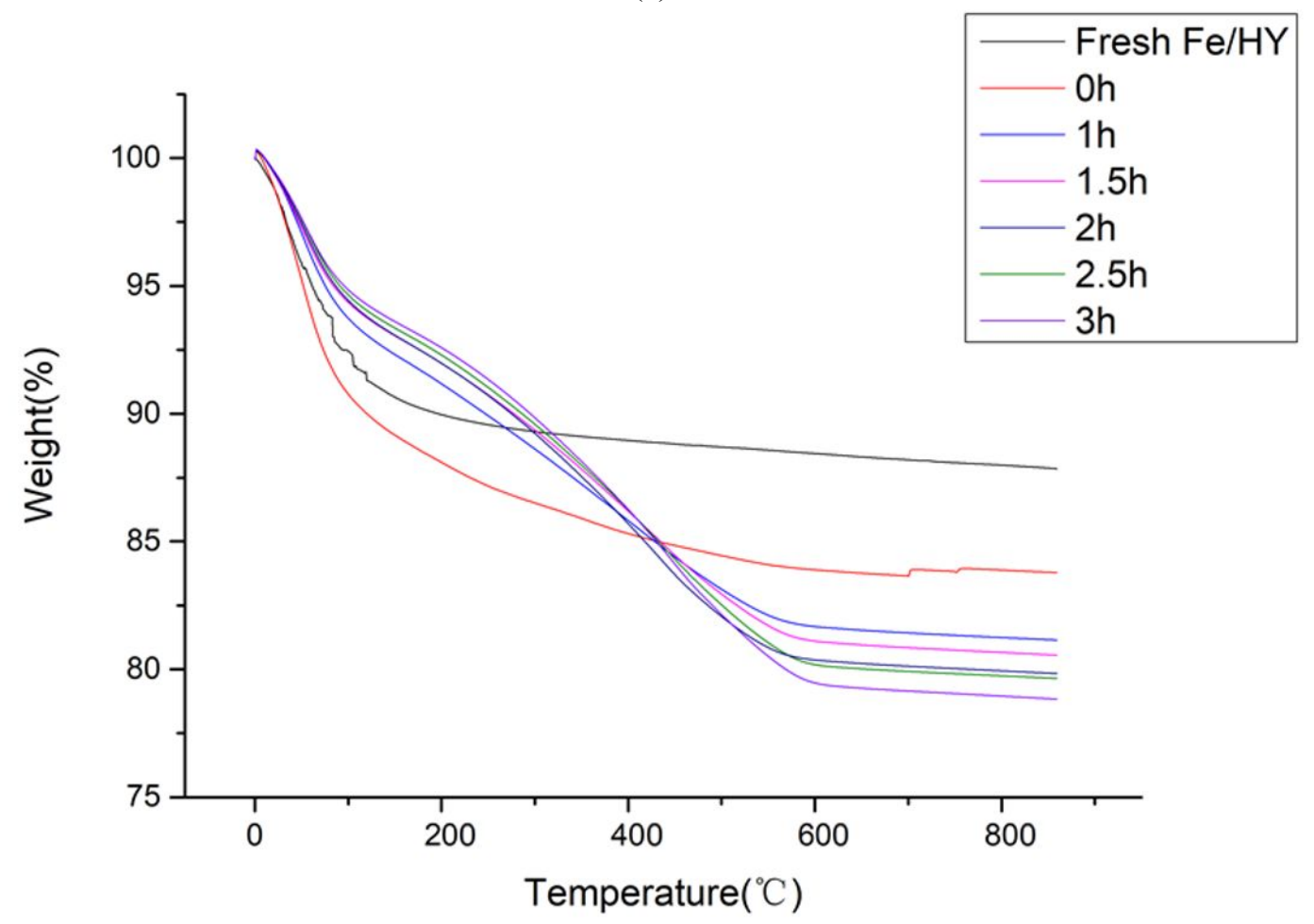

(b)

Figure S3. Thermogravimetric results of fresh and coked catalysts at $180{ }^{\circ} \mathrm{C}$ (a) and $200^{\circ} \mathrm{C}(\mathrm{b})$ in air. 
Table S1. ${ }^{a} \quad$ Supplementary Fe/HY-catalyzed Kinetic Parameters in simulation ${ }^{1}$

\begin{tabular}{ccccc}
\hline Parameter & Reaction & Reaction & Reaction & Reaction \\
& 1 & 2 & 3 & 4 \\
\hline $\mathrm{E}_{\mathrm{a}}\left(\mathrm{kJ} \mathrm{mol}^{-1}\right)$ & 64.0 & 75.7 & 60.8 & 70.2 \\
$\mathrm{~A}\left(\mathrm{~min}^{-1}\right)$ & $39.9 \times 10^{4}$ & $69.3 \times 10^{5}$ & $22.3 \times 10^{4}$ & $27.0 \times 10^{5}$ \\
$\mathrm{R}^{2}$ & 96.9 & 96.7 & 99.4 & 99.5 \\
\hline
\end{tabular}

Table S2. ${ }^{\text {a }} \quad$ Supplementary non-catalyzed Kinetic Parameters in simulation ${ }^{2}$

\begin{tabular}{ccccc}
\hline Parameter & Reaction & Reaction & Reaction & Reaction \\
& 1 & 2 & 3 & 4 \\
\hline $\mathrm{E}_{\mathrm{a}}\left(\mathrm{kJ} \mathrm{mol}^{-1}\right)$ & 108.03 & 135.71 & 89.28 & 108.91 \\
$\mathrm{~A}\left(\mathrm{~min}^{-1}\right)$ & $5.06 \times 10^{9}$ & $4.29 \times 10^{12}$ & $89.28 \times 10^{6}$ & $2.62 \times 10^{6}$ \\
$\mathrm{R}^{2}$ & 99.4 & 97.0 & 98.6 & 99.3 \\
\hline
\end{tabular}

a The corresponding kinetic models in Table S1 and S2 were proposed based on the pseudo homogeneous first-order approach. All the reactions were irreversible reactions. 


\section{Reference}

1. Ramli, N. A. S.; Amin, N. A. S., Kinetic study of glucose conversion to levulinic acid over Fe/HY zeolite catalyst. Chemical Engineering Journal 2016, 283, 150-159.

2. Jing, Q.; LÜ, X., Kinetics of Non-catalyzed Decomposition of Glucose in High-temperature Liquid Water. Chinese Journal of Chemical Engineering 2008, 16 (6), 890-894. 